\title{
SIMULATION OF TURBULENT FLOW THROUGH TARBELA DAM TUNNEL 3
}

\author{
Muhammad AbId ${ }^{1}$, Adam Aslam Noon ${ }^{1}$ ANd Hafiz Abdul WaJID ${ }^{2}$ \\ ${ }^{1}$ Faculty of Mechanical Engineering, GIK Institute of Engineering Sciences and Technology, \\ Topi, KPK, Pakistan \\ ${ }^{2}$ Department of Mathematics, COMSATS Institute of Information Technology, Lahore, Pakistan
}

E-mails: abid@giki.edu.pk,maangiki@yahoo.com,hadulwajid@yahoo.com

\begin{abstract}
Tarbela dam is one of the largest earth filled dam in the world. The inflow of sediments in the Tarbela reservoir has resulted in reduction in water storage capacity. During recent years, a reasonable increase of sediment particles in the tunnel has been observed. This is damaging to the tunnels, power generating units and is a severe threat to the plant equipment. To the authors knowledge, to-date no comprehensive simulation studies have been performed for flooding in the reservoir or turbulent flows in the tunnels. In this paper, turbulent flow in Tunnel 3 of the Tarbela Dam is analyzed using Reynolds Stress Model with and without considering the effect of sediments particle. Results are presented for three different water heads in the reservoir i.e. considering summer, winter and average seasons and for one-way and two-way/full coupling for sediments particle tracking/deposition. The effect of cavitation erosion and damage to the tunnels due to erosion is investigated and results are compared with the experimental erosion results for similar geometries and are found to be in good agreement. Sediments particulate analysis is also performed for the validation of the samples collected from WAPDA (water and power development authority). Moreover, pressure, velocity and erosion rate results are discussed to get complete behavior of the turbulent flow of water in the tunnel.
\end{abstract}

KEYWORDS: Tunnel, Turbulent flow, Sediment particles, Turbulent modeling, Cavitations, Erosion

\section{INTRODUCTION}

Tarbela Dam comprises of six tunnels, three of which are used for power generation and three for irrigation purposes. Moreover there are four outlets of the tunnel used for power generation and four pressure relief valves. Further details of this tunnel are given in Table $1[1,2]$. During recent years, a reasonable increase of sediment particles in the tunnel has 
been observed. This is damaging to the tunnels, power generating units and is a severe threat to the plant equipment. To the authors knowledge, to-date no comprehensive simulation studies have been performed for flooding in the reservoir or turbulent flows in the tunnels. In this paper, turbulent flow in Tunnel 3 of the Tarbela Dam is analyzed using Reynolds Stress Model with and without considering the effect of sediments particle. Results are presented for three different water heads in the reservoir i.e. considering summer, winter and average seasons and for one-way and two-way/full coupling for sediments particle tracking/deposition. The effect of cavitation erosion and damage to the tunnels due to erosion is investigated and results are compared with the experimental erosion results for similar geometries and are found to be in good agreement. The studies performed in this paper are carried out on a large structure, the tunnel lengths and diameters are in meters. It makes this research work unique and different from previous studies cited as references in the paper. In addition, Sediments particulate analysis is also performed for the validation of the samples collected from WAPDA (water and power development authority). Moreover, pressure, velocity and erosion rate results are discussed to get complete behavior of the turbulent flow of water in the tunnel. In the related work, Alamgir et al. [3] have investigated the particle deposition and suspension in a horizontal pipe flow. The deposition was studied as a function of particle diameter, density and velocity of fluid. The lighter particles were found to remain suspended with homogeneous distribution. The larger particles clearly showed deposition near the bottom of the wall. In our work the diameter of the flow passage is very large, the results show the dependance of velocity, pressure and erosion rate density on the passage diameter. Xianghui et al. [4] presented a computational fluid dynamics (CFD)-based erosion prediction model and its application to oilfield geometries specifically elbows and plugged tee geometries. This comprehensive procedure consists of three major components: flow simulation, particle tracking, and erosion calculation. The analysis procedure is taken from this study but cavitation analysis procedure is different from this. Gary [6] explains the Lagrangian approach for particle tracking, authors have used the same approach in one way coupling but Eularian approach is used for two way coupling. Hari [7] explains the role of different forces when a solid particle passes through a fluid, but in our case the rotational force is ignored.

Table 1: Details of Tunnel 3 [1, 2].

\begin{tabular}{|l|c|}
\hline \multicolumn{1}{|c|}{ Parameter } & Value \\
\hline Length $(\mathrm{m})$ & 1394.98 \\
\hline Inlet Elevation $(\mathrm{m})$ & 360.43 \\
\hline Inlet Diameter $(\mathrm{m})$ & 13.72 \\
\hline Outlet Branch Diameter $(\mathrm{m})$ & 7.32 \\
\hline Outlet Elevation $(\mathrm{m})$ & 337.11 \\
\hline High Head $(\mathrm{kPa})$ & 1096.66 \\
\hline Medium Head $(\mathrm{kPa})$ & 723.65 \\
\hline Low Head $(\mathrm{kPa})$ & 344.46 \\
\hline Average volume flow rate $\left(\mathrm{m}^{3} / \mathrm{s}\right)$ & 1318.05 \\
\hline Material of the steel liner & $5.4-6.35$ \\
\hline Steel liner thickness (cm) & 4 \\
\hline Quantity of turbines for power generation (Nos) & 432 \\
\hline Capacity of each Turbine (MW) & 1728 \\
\hline For four Turbines (MW) & High strength low alloy steel (A-441) \\
\hline
\end{tabular}




\section{THEORY}

\subsection{Turbulent Flow and Particle Tracking}

For flows with large, rapid, extra strains and flows with strong acceleration or retardation in the tunnel, we use second-moment closure model or Reynolds stress model (RSM) as it can reproduce anisotropy of the flow in the turbulent boundary layer. Coefficients of this model are given in Table 2 [3].

Table 2: RSM Model Constants [3].

\begin{tabular}{|c|c|}
\hline $\mathrm{C}_{\mu}$ & 0.09 \\
\hline$\sigma_{\mathrm{k}}$ & 1.0 \\
\hline $\mathrm{C}_{\mathrm{s} 1}$ & 1.8 \\
\hline $\mathrm{C}_{\mathrm{s} 2}$ & 0.6 \\
\hline $\mathrm{C}_{1 \varepsilon}$ & 1.44 \\
\hline $\mathrm{C}_{2 \varepsilon}$ & 1.92 \\
\hline$\sigma_{\varepsilon}$ & ------ \\
\hline
\end{tabular}

For analysis of turbulent flow in the tunnel, we divide variables into time-averaged part and fluctuating part. Governing equations of continuity and momentum for flow employed in CFX-11 are given in equations 1 and 2 respectively [4].

$$
\begin{aligned}
& \frac{\partial \rho}{\partial t}+\nabla \cdot\left(\rho \overrightarrow{U_{c}}\right)=0 \\
& \frac{\partial\left(\rho \overrightarrow{U_{c}}\right)}{\partial t}+\nabla \cdot\left(\rho \overrightarrow{U_{c}} \otimes \overrightarrow{U_{c}}\right)=\vec{B}+\nabla \cdot\left(-\rho \overrightarrow{u^{\prime} \otimes u^{\prime}}\right)+\sigma
\end{aligned}
$$

Here, $\rho \overline{u^{\prime} \otimes u^{\prime}}$ is the Reynolds stress; and stress tensor $\sigma$ is given by:

$$
\sigma=-\frac{p}{\rho} I+\frac{\mu}{\rho}\left[\nabla \overrightarrow{U_{c}}+\left(\nabla \overrightarrow{U_{c}}\right)^{T}\right]
$$

For sediments particle deposition in turbulent flow, we use Lagrangian particle transport and Eulerian-Eulerian multiphase approaches [5], using a RANS framework.

One-way and two-way/full coupling options are used depending upon the value of $\beta$, which is defined as the ratio of the particulate mass per unit volume flow to the fluid mass per unit volume flow and is given by;

$$
\beta=\frac{r_{p} \rho_{p}}{r_{f} \rho_{f}}
$$


where, $\beta=0.2$ is the threshold value [6]. One-way coupling is valid for volume concentration up to $14.86 \%$ and simply predicts particle paths during post-processing based on the flow field without affecting the flow field (i.e. particles are assumed not to interact with each other). In two way/full coupling particles exchange momentum with continuous phase, allowing the continuous flow to affect the particles and vice versa.

Generally, a small, rigid spherical particle entrained in the turbulent pipe flow encounters many forces [7], such as drag and lift forces, buoyancy force, rotation force and turbulence force etc and has the following general form

$$
\frac{d U_{p}}{d t} \equiv F_{D}\left(U_{c}-U_{p}\right)+g_{x}\left(\frac{\rho_{p}-\rho}{\rho_{p}}\right)+F_{x}
$$

where, $F_{x}$ represents additional forces in the particle force balance that can be used under special circumstances. Moreover, $g_{x}$ is the force of gravity. For present study we have ignored the force of gravity on the particle, it is important to note that for most cases the gravitational acceleration is taken zero. One can include the gravity force, but should define the magnitude and direction of the gravity vector. Virtual mass force which is used to accelerate the fluid surrounding the particle is also ignored alongwith additional forces arise due to the pressure gradient in the fluid and due to rotation of the reference frame. Later forces are important only when fluid is flowing in a rotating frame. Another force which is not related for our model is produced because of temperature gradient effecting small particles suspended in a gas. This phenomenon is known as thermophoresis. For micro particles, the effects of Brownian motion is optional and if required can be included in the additional force term. Therefore, the governing particle equation of motion takes the following form:

$$
\frac{d \vec{U}_{p}}{d t}=F_{D}\left(\overrightarrow{U_{c}}-\overrightarrow{U_{p}}\right)
$$

where drag force per unit mass is expressed as:

$$
F_{D}=\frac{1}{\tau_{p}} C_{D} \frac{\mathrm{Re}_{p}}{24}
$$

with $\tau_{p}$ as the particle response time and is given by:

$$
\tau_{p}=\frac{\rho_{p} d_{p}^{2}}{18 \mu}
$$

Furthermore $R e_{p}$ is the particle Reynolds number based on the relative velocity between the particles and carrier phase given by

$$
\operatorname{Re}_{p}=\frac{d_{p}\left(\vec{U}-\overrightarrow{U_{p}}\right)}{v}
$$


Moreover $C_{D}$ is the drag coefficient and is used in CFX by the Schiller Naumann correlation. Schiller Naumann correlation is derived for flow of a single spherical particle and is valid in the dilute limit of small solid phase volume fractions for one-way coupling with a coefficient of 0.1 . Particle transport drag coefficient correlation is used in twoway/full coupling phenomena with a coefficient of 0.44 [8].

\subsection{Erosion Estimation and Surface Damage}

Sediment erosion phenomenon is highly complicated and a wide range of factors contribute to erosion severity [9]. Analysis is done using ANSYS CFX to determine the erosion rate under turbulent flow in the tunnel for different heads, and variation of particles concentration. In ANSYS CFX, only Finnie and Tabakoff erosion models are available. Tabakoff model provides more scope for customization with its larger number of input parameters but presence of large number of coefficients in the model can become a source of errors in the numerical scheme [10]. We therefore use simplified erosion model of Finnie with Langrangian particle tracking and Eulerian-Eulerian multiphase approaches.

We have used the following expression for erosion rate density:

$$
\begin{gathered}
E=k \cdot V_{p}^{2} \cdot f(\gamma) \quad \text { with } \\
f(\gamma)=\frac{1}{3} \cos ^{2}(\gamma) \quad \text { if } \gamma>18.26^{\circ} \\
f(\gamma)=\sin (2 \gamma)-3 \sin ^{2}(\gamma) \text { if } \gamma<18.26^{\circ}
\end{gathered}
$$

In ANSYS CFX, implementation of overall erosion rate at each point on the surface is calculated by multiplying $E$ with mass flow carried by the Langrangian particle impacting the surface, and then summing for all particles. This ultimately leads to an erosion rate density variable.

Deformation wear occurs when repeated particle impacts at high impact angles and plastically deform surface layers of the material, eventually causing material loss through surface fragmentation. Cutting wear occurs due to particle impacts at small angles, with a scratch or cut being formed on the surface if the shear strength of the material is exceeded. The other critical factor affecting wear is the particle impact velocity, with both cutting and deformation wear being proportional to impact velocity raised to a power $n$ determined through physical tests. In general $n$ varies between 2 and 3 depending on both the surface and particle materials. The value of $k$ is set to 1 which has been determined experimentally [11].

The total erosion rate at a particular point on a surface is measured by summing the contributions due to the deformation and cutting mechanisms and depends on the properties of the material, with deformation wear being more significant for hard or brittle materials and cutting wear being more significant for softer or ductile materials. For standard commercial grade steels, as used in most of the bend surfaces, peak erosion rates 
have been measured to occur at impact angles of $25-30^{\circ}$ [12], indicating that cutting wear dominates.

\subsection{Cavitation Erosion}

Cavitation is a phenomenon of formation of vapor bubbles in low pressure regions and collapse in high pressure regions. High pressure is produced and metallic surfaces are subjected to high local stresses. It is a phenomenon which manifests itself in the pitting of the metallic surfaces of tunnel parts because of the formation of cavities. The most critical location is the S-bend at the middle of the tunnel where pressure falls well below the vapour pressure i.e; $750 \mathrm{~Pa}$ in one way coupling and $450 \mathrm{~Pa}$ in two way/full coupling. The tendency for a flow to cavitate is characterized by the pressure coefficient or cavitation number, given by [13]:

$$
C a=\frac{\left(p_{r}-p_{v}\right)}{0.5 \times \rho U_{c}^{2}}
$$

\subsubsection{Cavitation Models}

Different models have been proposed for cavitation phenomenon in literature, but here we consider the followings:

The Rayleigh-Plesset equation provides the basis for the rate equation controlling vapour generation and condensation. The Rayleigh-Plesset equation describing the growth of a gas bubble from a liquid is given by:

$$
R_{B} \frac{d^{2} R_{B}}{d t^{2}}+1.5\left(\frac{d R_{B}}{d t}\right)^{2}+\frac{2 \sigma}{\rho_{f} R_{B}}=\frac{p_{v}}{\rho_{f}}
$$

The Homogeneous model can be viewed as a limiting case of Eulerian-Eulerian multiphase flow in which the inter phase transfer rate is very large. This model is used in highly dispersed flows with one gas phase and the other dispersed liquid droplet phase.

For the present study we use Rayleigh Plesset cavitation model which requires the model parameters given in Table 3 [14].

Table 3: Rayleigh Plesset cavitation model [14].

\begin{tabular}{|c|c|}
\hline Parameter & Value \\
\hline$P_{s}$ & $4240 \mathrm{~Pa}$ \\
\hline$F_{\text {cond }}$ & 0.01 \\
\hline$F_{\text {vap }}$ & 50 \\
\hline
\end{tabular}




\begin{tabular}{|c|c|}
\hline Pr & 1000 \\
\hline$r_{\text {nuc }}$ & $5 \mathrm{e}-4$ \\
\hline $\mathrm{Rf}_{\mathrm{f}}$ & 0.25 \\
\hline $\mathrm{R}_{\mathrm{B}}$ & $1 \mu \mathrm{m}$ \\
\hline
\end{tabular}

\section{MODELING AND ANALYSIS}

Modeling of tunnel is done in Pro-Engineer software as per dimensions taken from [15] and is shown in Fig. 1. Tunnel model is then meshed in ICEM CFX with free mesh option using 1843803 tetrahedral elements as shown in Fig. 2.

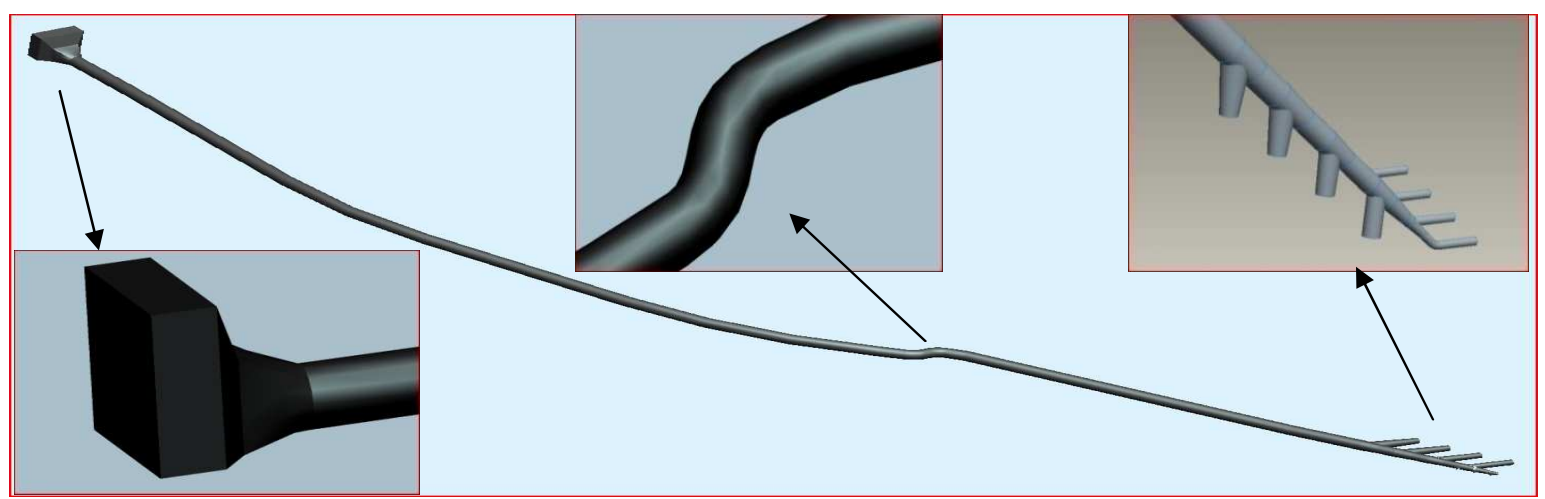

Fig. 1: Tunnel Model.

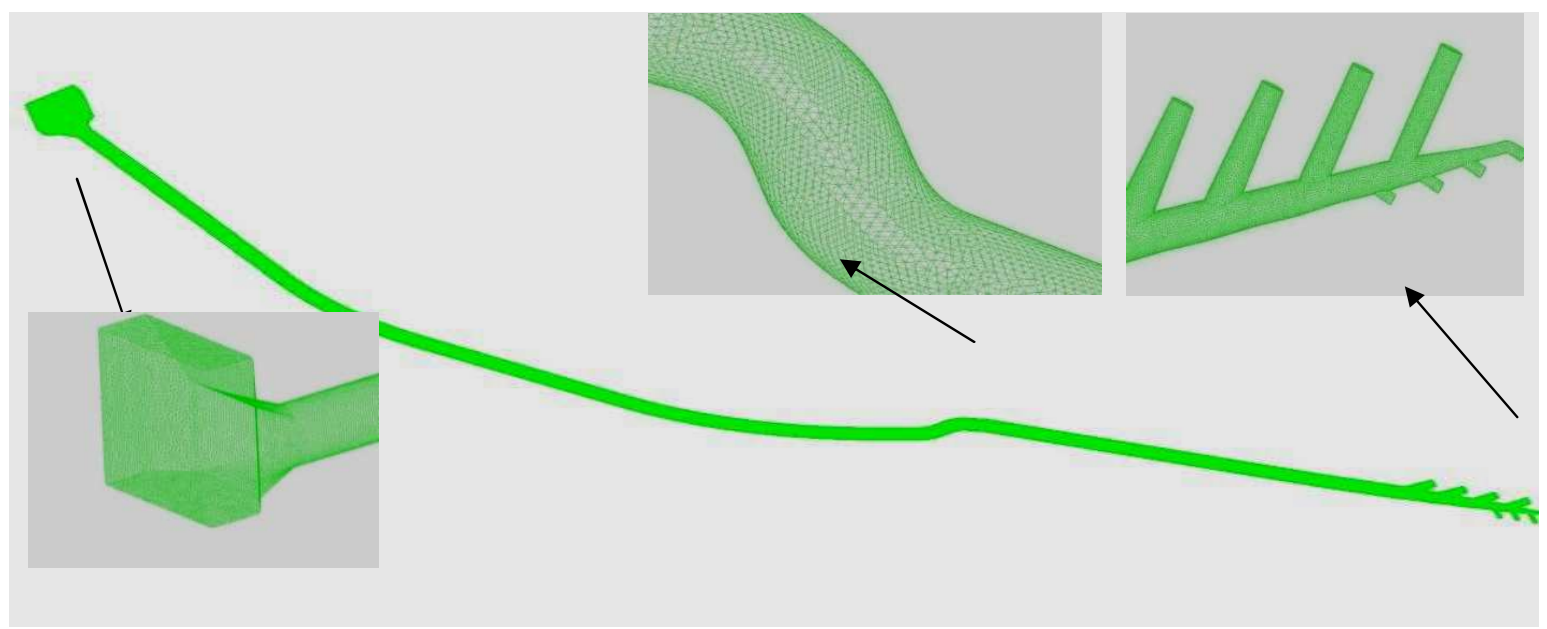

Fig. 2: Tunnel Mesh 
Meshed model is imported into ANSYS CFX for detailed analysis. The particles are assumed to be randomly distributed at the inlet. Particles injected at the inlet are proportional to the mass flow rate of water flowing into the tunnel. The sediment particles volume fraction is only $0.007 \%$ at the high head during months of July till September, which increases to $6.1 \%$ at the minimum head level during March till June. These fractions fall in the case of one-way coupling. The samples collected and analyzed in February 2010 show that the concentration of sediment particle has increased to $19.6 \%$ which lies in the two-way/full coupling region as it crosses the threshold value of $14.86 \%$ [6]. The analysis reveals that the continuous flow changes to dispersed flow and flow field is now affected by the sediment particles, which is more critical for the damage caused by erosion. Standard no-slip wall functions were applied at all solid surfaces for the fluid phase. Furthermore, coefficient of restitution for the particles were taken as 0.9 for parallel flow direction and 1.0 for perpendicular flow direction.

The effect of cavitation erosion is analyzed at the S-bend of tunnel of $200 \mathrm{~m}$ length. The velocity boundary conditions of $38.51 \mathrm{~m} / \mathrm{s}$ and $32.09 \mathrm{~m} / \mathrm{s}$ are specified at the inlet and outlet of the S-bend portion.

During analysis, pressure relief valves are excluded in the geometry which might affect the water velocities and pressures at different locations of the tunnel. Boundary and initial conditions applied are listed in Table 4. Zero pressure is specified at the tunnel outlet being exposed to the atmosphere. The list of other input parameters is given in Table 5.

Table 4: Boundary conditions and Initial conditions.

\begin{tabular}{|c|c|c|c|}
\hline & Type & Head & Value \\
\hline \multirow{3}{*}{$\begin{array}{l}\text { Boundary Coditions } \\
\text { (BCs) }\end{array}$} & \multirow{3}{*}{ Pressure $(\mathrm{P}) \mathrm{kPa}$} & High & 1096.66 \\
\hline & & Medium & 723.65 \\
\hline & & Low & 344.46 \\
\hline \multirow{3}{*}{$\begin{array}{l}\text { Initial Conditions } \\
\text { (ICs) }\end{array}$} & \multirow{3}{*}{ Velocity (V) $\mathrm{ms}^{-1}$} & Low & 0.563 \\
\hline & & Medium & 0.930 \\
\hline & & High & 1.143 \\
\hline
\end{tabular}

Table 5: Input parameters used in ANSYS CFX.

\begin{tabular}{|c|l|l|c|}
\hline Sr \# & \multicolumn{1}{|c|}{ Parameter } & \multicolumn{1}{c|}{ Details } & Numerical numbers \\
\hline 1 & Mass flow rate & $\begin{array}{l}\text { Sediment particles mass flow rate at } \\
\text { high head }\end{array}$ & $5.63 \times 10^{-5} \mathrm{~kg} / \mathrm{s}$ \\
\hline 2 & Erosion model & Finnie & $\mathrm{k}=1.0$ and $\mathrm{n}=2.0$ \\
\hline 3 & Wall roughness & At the wall & $0.2 \mathrm{~mm}$ \\
\hline 4 & $\begin{array}{l}\text { Particles } \\
\text { injection }\end{array}$ & Uniform injection at the tunnel inlet & $\begin{array}{c}6.1 \% \text { particles for one-way } \\
\text { coupling and more than } 15 \% \\
\text { particles for full coupling }\end{array}$ \\
\hline 5 & $\begin{array}{l}\text { Restitution } \\
\text { coefficients }\end{array}$ & $\begin{array}{l}\text { Parallel and perpendicular based on } \\
\text { impact and rebound velocities }\end{array}$ & 0.9 and 1.0 \\
\hline
\end{tabular}




\begin{tabular}{|c|l|l|c|}
\hline 6 & Drag force & $\begin{array}{l}\text { Schiller and Naumann correlation } \\
\text { for the evaluation of drag } \\
\text { coefficient for one-way coupling }\end{array}$ & 0.1 \\
\cline { 3 - 4 } & $\begin{array}{l}\text { Particle transport drag coefficient } \\
\text { for two-way coupling }\end{array}$ & 0.44 \\
\hline 7 & $\begin{array}{l}\text { Numerical } \\
\text { Scheme }\end{array}$ & Specified blend factor & $0 \sim 1$ \\
\hline 8 & $\begin{array}{l}\text { Particle } \\
\text { integration }\end{array}$ & Tracking distance and time & $1394.98 \mathrm{~m}$ and $300 \mathrm{~s}$ \\
\hline
\end{tabular}

Navier-Stokes equations for mass, momentum and fluid turbulence are solved with the commercial code CFX-11 using the finite volume technique. Convection terms in momentum equations are discretized using a simple high resolution advection scheme.

\subsection{Mesh Sensitivity Analysis}

The purpose of mesh senstivity analysis is to determine the minimum grid resolution required to generate a solution that is independent of the grid used. Starting with a coarse grid the number of cells was increased in the region of interest until the solution from each grid was unchanged for successive grid refinements.

Mesh sensitivity analysis was performed using the following methodology:

1. Meshes of four different element sizes $(2,3,5$ and 7$)$ were generated using ANSYS ICEM.

2. Boundary conditions were applied to all these four meshes.

3. Transient analysis was performed for all the four meshes.

4. Final residuals were compared for differences in comparison for changes in mesh sizes.

All the results were compared with a mesh size of 3 as the standard. We have evaluated the effect of further refining the mesh to a mesh size of 2 and also coarsening it to a mesh size of 5. The mesh senstivity statistics are tabulated in Table 6 .

Results of important variables such as velocity, pressure and erosion rate density were also taken at a particular point in the tunnel defined by the coordinates $(793,1$, and -44$)$ and are given in Table 7.

Table 6: Mesh senstivity statistics.

\begin{tabular}{|c|c|}
\hline Mesh Size & Number of Elements \\
\hline 2 & 450,000 \\
\hline 3 & 300,000 \\
\hline 4 & 225,000 \\
\hline 5 & 180,000 \\
\hline
\end{tabular}


Table 7: Variable values for Tunnel 2 at four different mesh sizes.

\begin{tabular}{|c|c|c|c|c|}
\hline Variables & 2 & 3 & 5 & 7 \\
\hline Velocity (V) $\mathrm{ms}^{-1}$ & 72.06 & 72.25 & 75.34 & 76.44 \\
\hline Pressure $(\mathrm{P}) \mathrm{kPa}$ & 104.5 & 105 & 107 & 109 \\
\hline $\begin{array}{l}\text { Erosion rate density } \\
\text { (E) } \times 10^{-5} \mathrm{kgs}^{-1} \mathrm{~m}^{-2}\end{array}$ & 11.03 & 11.17 & 11.98 & 12.46 \\
\hline
\end{tabular}

\subsection{Equation Residual}

If the solution is "exact" then the residual is zero. Exact means that each of the relevant finite volume equations is satisfied precisely. The "residual" of an equation identifies by how much the left-hand side of the equation differs from the right-hand side at any point in space i.e; $\mathrm{Ax}-\mathrm{B}=$ residual.

The RMS residual is obtained by taking all of the residuals throughout the domain, squaring them, taking the mean, and then taking the square root of the mean. The peak values of residues, final residues as summarized in Table 8, convergence plots and important variables are compared for analysis performed on different mesh sizes for Tarbela Dam Tunnels.

Table 8: Final Residuals.

\begin{tabular}{|l|c|c|c|}
\hline \multirow{2}{*}{ Equations } & \multicolumn{3}{|c|}{ Element Mesh Size } \\
\cline { 2 - 4 } & $\mathbf{2}$ & $\mathbf{3}$ & $\mathbf{5}$ \\
\hline U-Mom & $7.89670 \mathrm{E}-03$ & $3.22020 \mathrm{E}-02$ & $7.49670 \mathrm{E}-02$ \\
\hline V-Mom & $7.34670 \mathrm{E}-01$ & $6.47095 \mathrm{E}-01$ & $5.34670 \mathrm{E}-01$ \\
\hline W-Mom & $3.16890 \mathrm{E}-02$ & $5.17751 \mathrm{E}-02$ & $6.16890 \mathrm{E}-02$ \\
\hline uu-RS & $1.98790 \mathrm{E}-02$ & $2.94994 \mathrm{E}-01$ & $1.98790 \mathrm{E}-01$ \\
\hline vv-RS & $3.46780 \mathrm{E}-01$ & $2.94975 \mathrm{E}-01$ & $1.46780 \mathrm{E}-01$ \\
\hline ww-RS & $4.12340 \mathrm{E}-01$ & $2.94995 \mathrm{E}-01$ & $3.12340 \mathrm{E}-01$ \\
\hline uv-RS & $2.87650 \mathrm{E}-02$ & $1.06785 \mathrm{E}-02$ & $2.37650 \mathrm{E}-02$ \\
\hline uw-RS & $8.94570 \mathrm{E}-04$ & $5.14682 \mathrm{E}-03$ & $7.94570 \mathrm{E}-04$ \\
\hline vw-RS & $5.23450 \mathrm{E}-02$ & $3.96466 \mathrm{E}-02$ & $1.23450 \mathrm{E}-02$ \\
\hline E-Diss.K & $2.84340 \mathrm{E}+02$ & $3.43782 \mathrm{E}+02$ & $4.84340 \mathrm{E}+02$ \\
\hline
\end{tabular}

\subsection{Convergence Plots}

Turbulence, mass and momentum plots for the residuals are shown in Fig. 3 and Fig. 4. The Mass and Momentum plot of the mesh size of 2 shows smoother convergences as compared to the other mesh however none of the meshes have shown large peaks of the 
residuals. This must be kept in mind that the mesh size of 2 takes far more computational power than the rest and still does not substantially improves the results.

The turbulence plot of the mesh size of 2 again shows smoother convergences when compared to other meshes. This could be because of the finer mesh but the residual peak values do not justify the amount of computational time and power required for such fine mesh.

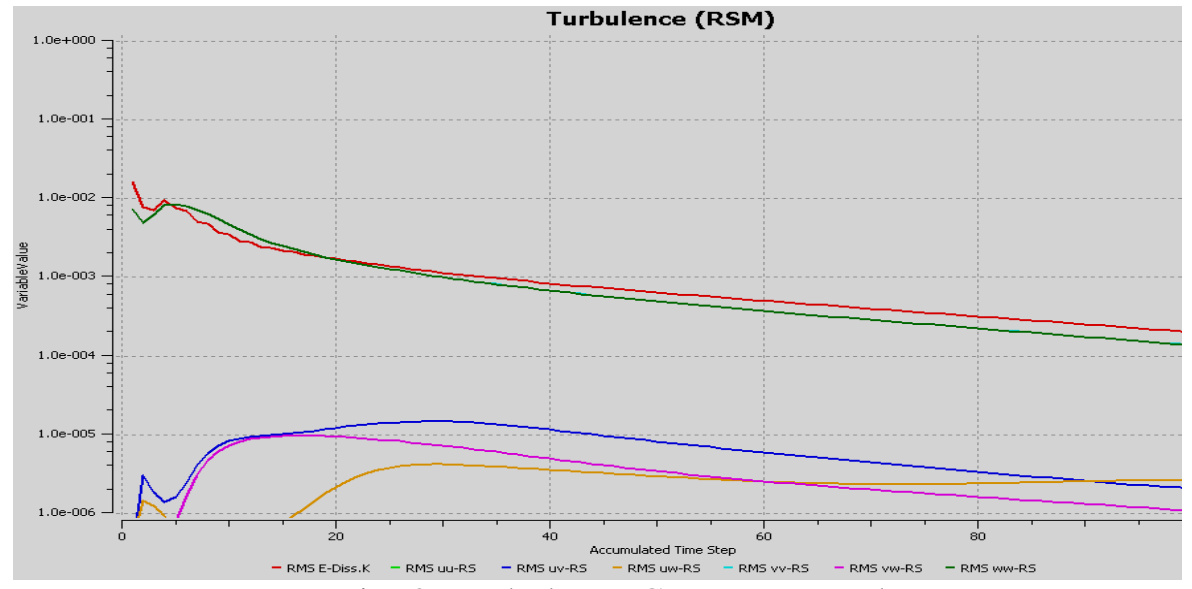

Fig. 3: Turbulence Convergence Plot.

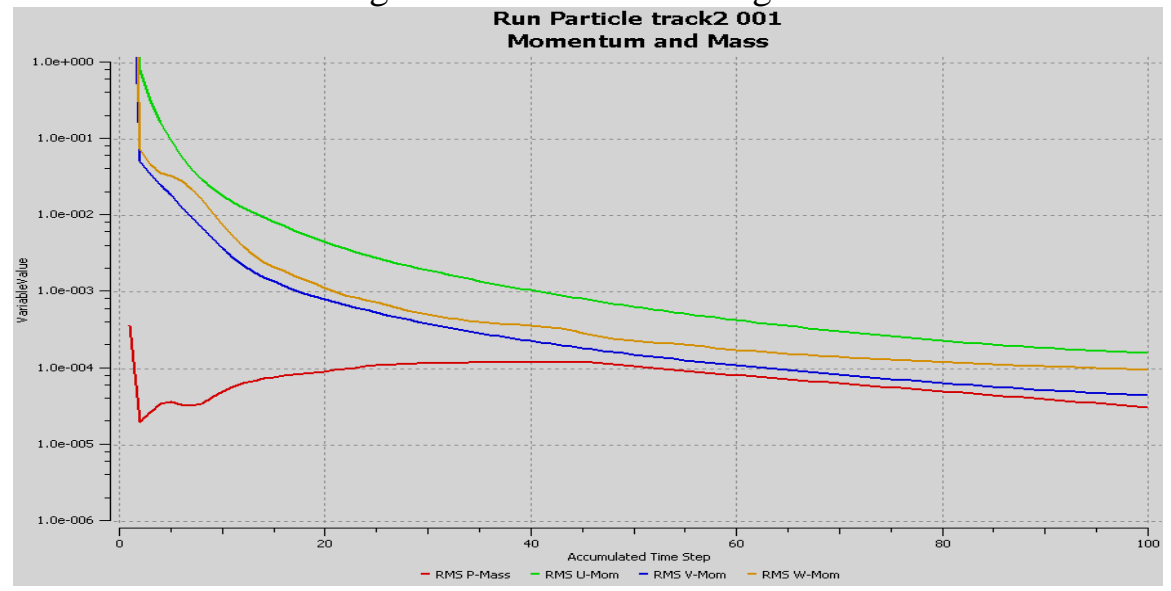

Fig. 4: Mass and Momentum Convergence Plot.

\section{RESULTS AND DISCUSSION}

The following sections are dedicated for complete analysis of results obtained using ANSYS CFX for velocity, pressure and erosion rate density for different coupling techniques and water heads. Specific attention is paid to critical region such as S-bend.

\subsection{One-way Coupling at All Heads with Sediment Particles}


For one way coupling maximum velocities of water $28.89,41.73$ and $51.34 \mathrm{~m} / \mathrm{s}$ are measured at low, medium and high head of water respectively at the S-bend. Velocities are $7.23,10.44$ and $12.84 \mathrm{~m} / \mathrm{s}$ at the inlet section. Velocity increases to $21.67,26.40$ and 32.09 $\mathrm{m} / \mathrm{s}$ when the water flow is fully developed at $150 \mathrm{~m}$ from the vertical section at low, medium and high water head respectively. Velocity increases abruptly at the outlet branches due to reduction in their crossectional area. Moreover, maximum pressures of water $346.70,728.10$ and $1103 \mathrm{kPa}$ are measured at the inlet section where the velocity was minimum at low, medium and high water heads respectively. Minimum pressures $1.75,4.50$ and $0.75 \mathrm{kPa}$ are measured at the $\mathrm{S}$-bend where the velocity has it highest value respectively at low, medium and high water heads. Pressure decreases abruptly at the main branch and at the outlet branches due to increase in the velocity. Furthermore, higher erosion rate densities $11.75 \times 10^{-5}, 8.13 \times 10^{-5}, 7.13 \times 10^{-5} \mathrm{kgs}^{-1} \mathrm{~m}^{-2}$ due to the sediment particles are measured at high, medium and low heads of water respectively at the inlet section are measured. The highest value of the erosion rate density is measured as $23.31 \times 10^{-5} \mathrm{kgs}^{-1} \mathrm{~m}^{-2}$ at the S-bend. It changes abruptly at the main branch and at the outlet branches due to the higher impact velocity and impact angle at these locations.

\subsection{Effect of Coupling at High Head}

In one way coupling increase in sediment particles concentration does not effect the velocity, pressure and erosion rate density, but in two way/full coupling the velocity of water deccreases to about $5 \%$ and pressure of water increases to $1 \%$ at high head. The small change in velocity and pressure values is due to the fact that the diameter of the tunnel is too large to effect the flow field completely. But the erosion rate density of tunnel wall increases to about $35 \%$ because of the higher number of sediment particles striking the tunnel walls with higher impact velocity at high head. The results are shown in Fig. 57 for one way coupling and Fig. 8-10 for two way/full coupling.

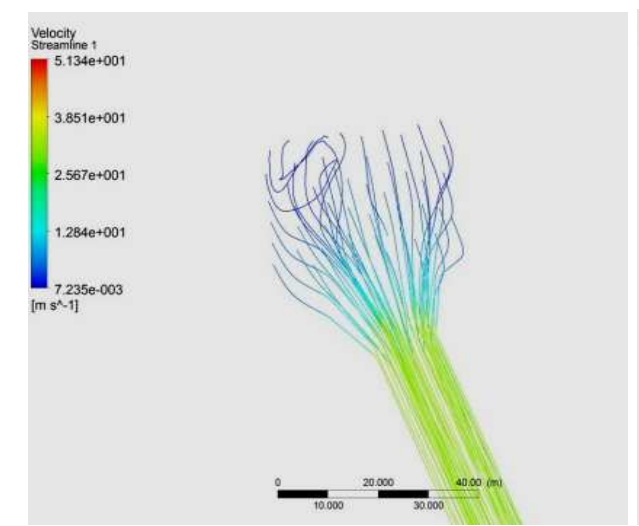

(a)

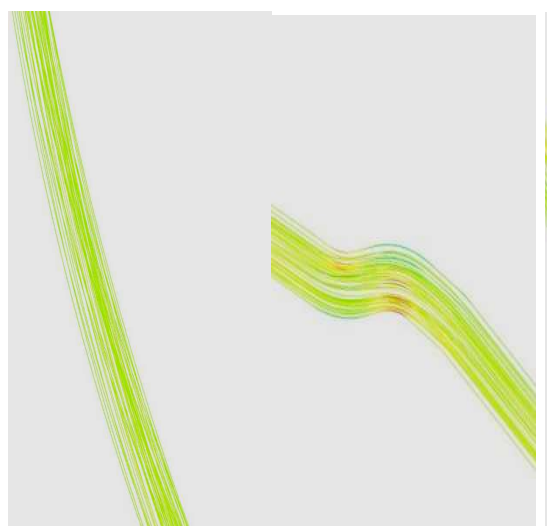

(b) (c)

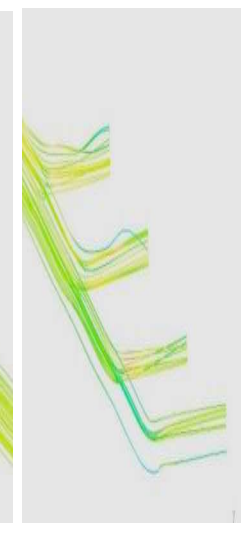

(d)

Fig. 5: One way coupling with sediments: Velocity profiles at high head at: (a) Inlet, (b) Straight portion, (c) S-bend, (d) Outlet branches. 


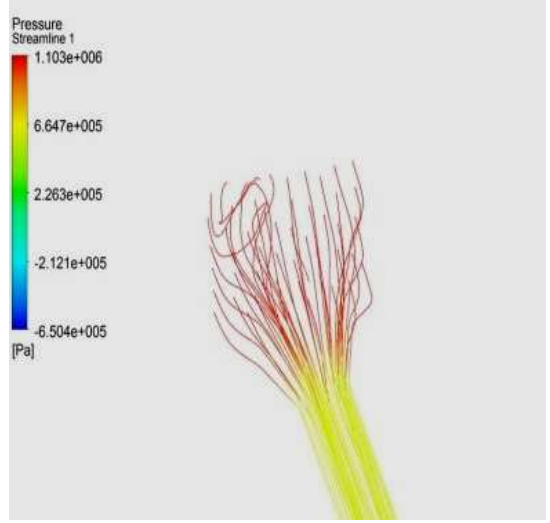

(a)

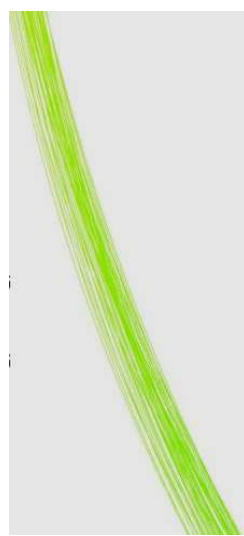

(b)

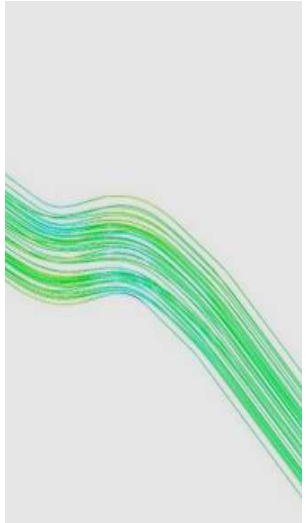

(c)

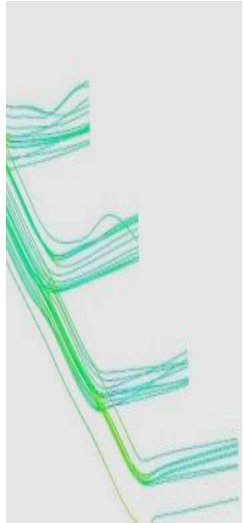

(d)

Fig. 6: One way coupling with sediments: Pressure profiles at high head at: (a) Inlet, (b) Straight portion, (c) S-bend, (d) Outlet branches.

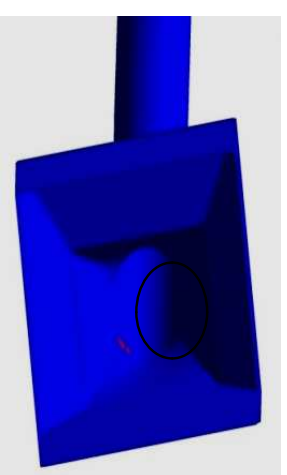

(a)

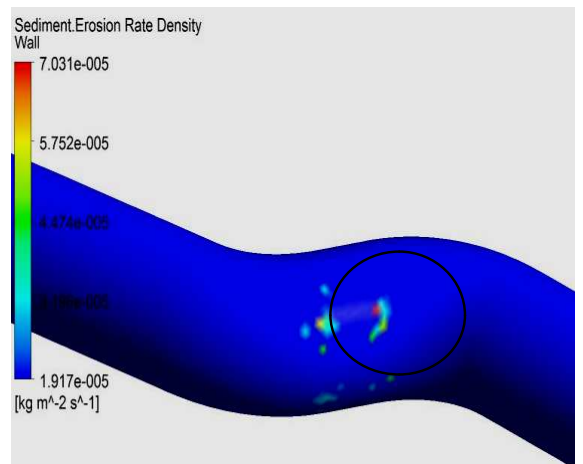

(b)

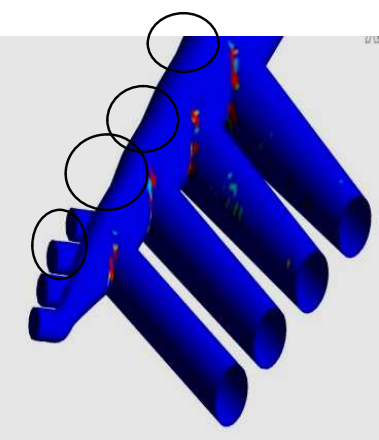

(c)

Fig. 7: One way coupling with sediments: Erosion rate density profiles at high head: (a) Inlet, (b) S-bend, (c) Outlet branches.

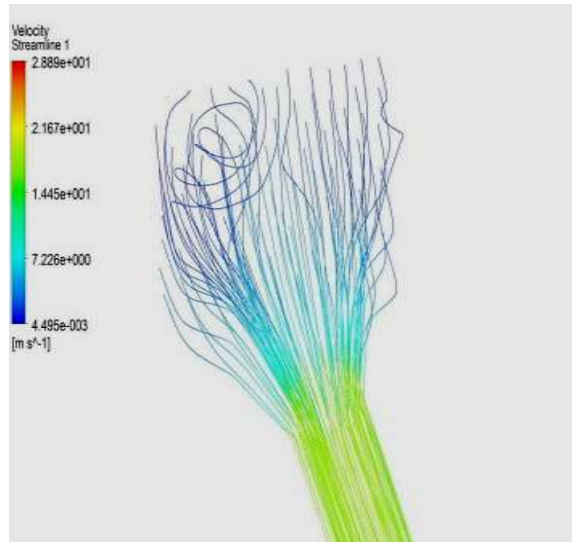

(a)

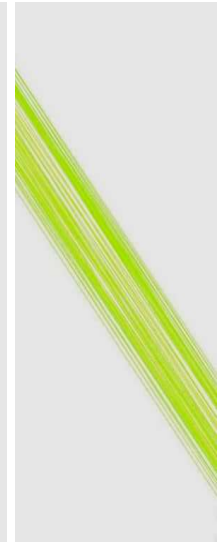

(b)

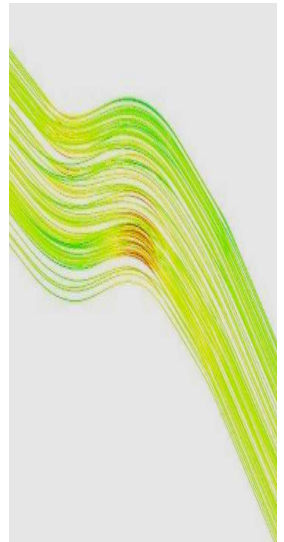

(c)

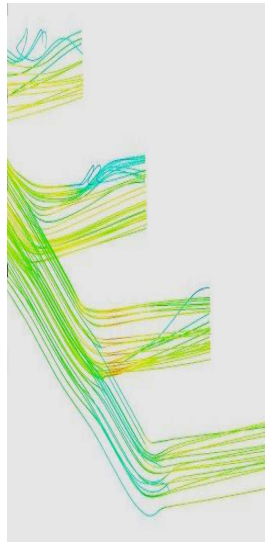

(d)

Fig. 8: Two way/full coupling with sediments: Velocity profiles at high head at: (a) Inlet, (b) Straight portion, (c) S-bend, (d) Outlet branches. 


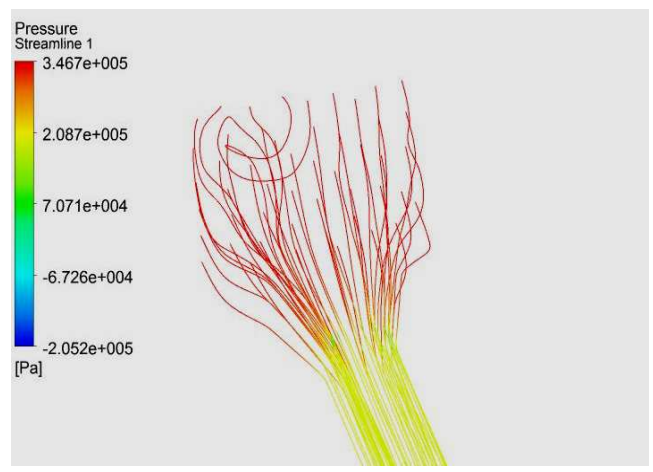

(a)

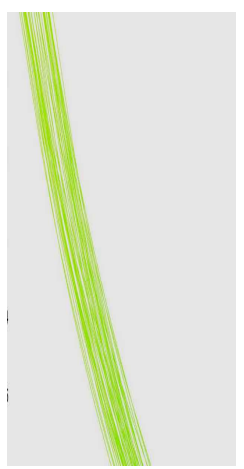

(b)

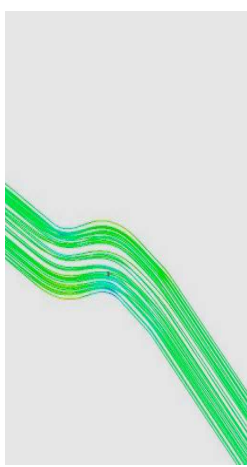

(c)

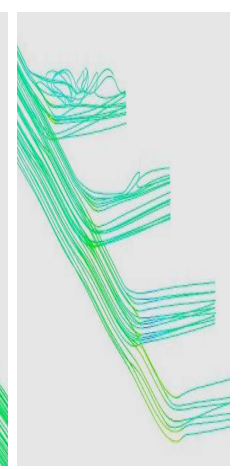

(d)

Fig. 9: Two way/full coupling with sediments: Pressure profiles at high head at: (a) Inlet, (b) Straight portion, (c) S-bend, (d) Outlet branches.

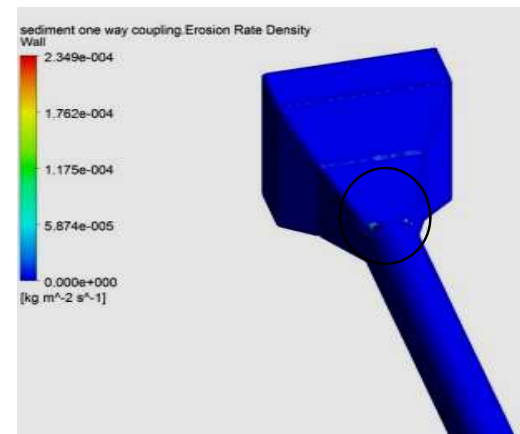

(a)

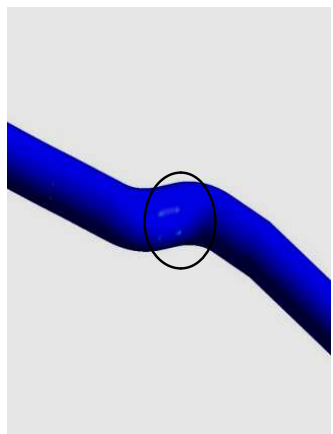

(b)

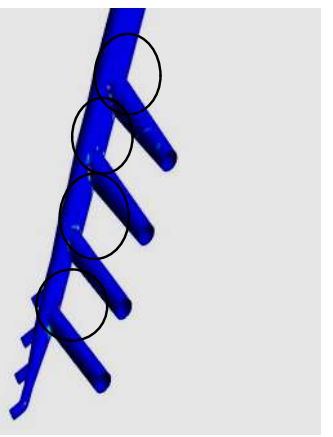

(c)

Fig. 10: Two way/full coupling with sediments: Erosion rate density profiles at high head: (a) Inlet, (b) S-bend, (c) Outlet branches.

The velocity, pressure and erosion rate density values will be effected significantly when the sediment particles concentration reaches to the threshold value of $14.86 \%$ as the sediment delta is moving towards the tunnel. This shows that the coupling phenomena is a function of tunnel diameter.

\subsection{Effect of Cavitation at High Head at S-bend}

The cavitation phenomenon is observed at the S-bend for tunnel 3 due to the lower local pressure then the vapour pressure at this critical location. The local pressure at $\mathrm{S}$ bend is $3.57 \mathrm{kPa}$ which is less than the vapour pressure of water i.e; $4.24 \mathrm{kPa}$ as shown in Fig. 11(a), therefore cavitation phenomena starts at this location. The water vapour volume fraction profile is shown in Fig. 11(b). The highest fraction of 0.795 is found at the inner periphery of the curvature of the S-bend. The low pressure at this location generates the bubble formation, when these bubbles enter the higher pressure region, they explode. As a 
result these bubble bursts causes the cavitaion erosion. The velocity profiles for water flow are discontinuous, i.e, water contents dominates the vapor contents at some portions and vice versa due to the mixing of water and water vapour phases (two phase flow) at S-bend as shown in Fig. 12.

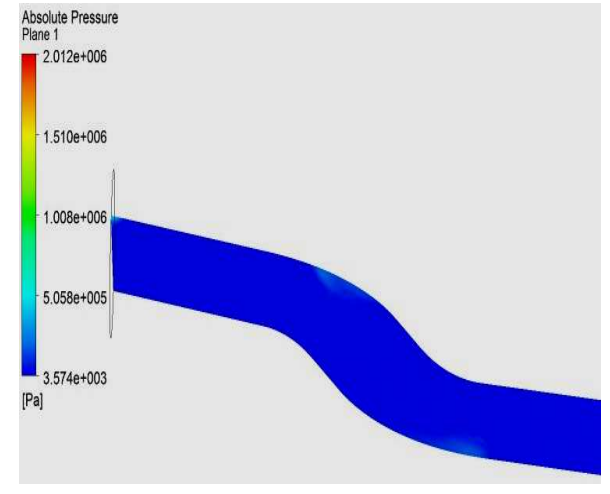

(a)

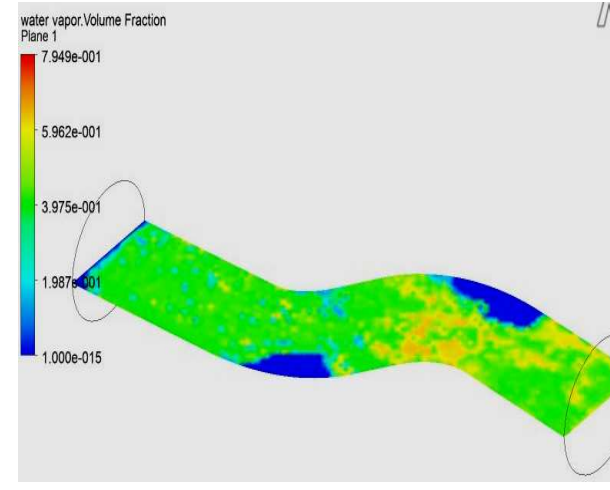

(b)

Fig. 11: Cavitation at S-bend surface: (a) Absolute pressure, (b) Water vapour volume fraction.

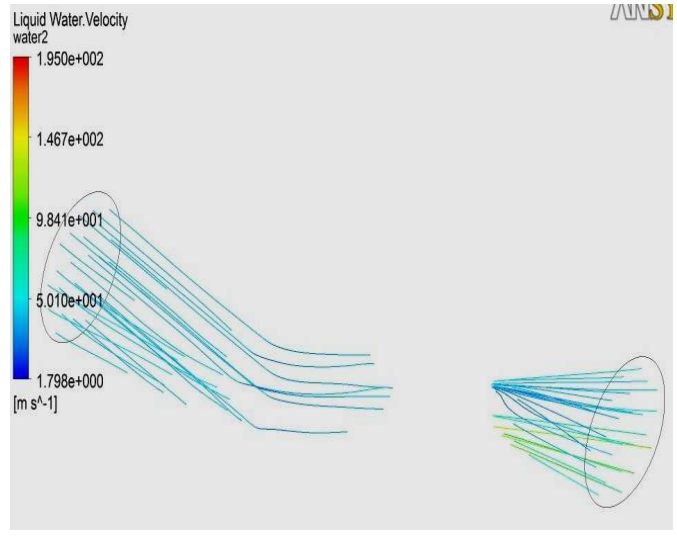

(a)

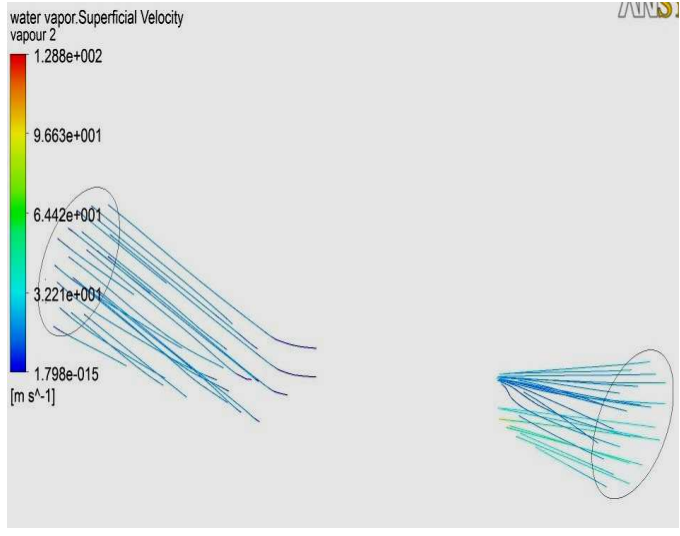

(b)

Fig. 12: Velocity profiles at S-bend for: (a) water, (b) water vapour.

\subsection{Effect of no Sedimentation at High Head}

Negligible increase of less than $2 \%$ in velocity and pressure is observed for the sediments particle flow. This demonstrates that the flow field is uneffected in one way coupling. Erosion in the tunnel for flow without the sediment particles is concluded due to the turbulent eddies [16] present at the walls of the tunnels at critical locations. 


\subsection{Critical Period for Erosion at Different Heads}

The velocity and pressure are maximum at high head in the months of July, August and September which impedes the erosion rate and is the most critical period. The medium head remains for five months i.e. April, May, June, October and November, when the velocity and pressure are observed moderate. The low head remains for four months i.e. December, January, February and March when the water velocity and pressure are minimum. The results are summarized in Table 9. Hence high head is crucial for erosion damage.

Table 9: Velocity, pressure and erosion rate density at all heads for Tunnel 3.

\begin{tabular}{|c|c|c|c|c|c|c|c|}
\hline Parameter & Head & $\begin{array}{c}\text { Inlet } \\
\text { Section }\end{array}$ & S-Bend & Branch 1 & Branch 2 & Branch 3 & Branch 4 \\
\hline \multirow{3}{*}{$\underset{\text { ms }^{-1}}{\text { Velocity }}(\mathbf{V})$} & Low & 6.56 & 28.89 & 28.90 & 29.74 & 27.25 & 24.88 \\
\hline & Med & 9.74 & 41.73 & 31.30 & 31.30 & 31.30 & 31.30 \\
\hline & High & 12.94 & 51.34 & 38.51 & 38.51 & 38.51 & 38.51 \\
\hline \multirow{3}{*}{$\begin{array}{c}\text { Pressure (P) } \\
\mathrm{kPa}\end{array}$} & Low & 438.7 & 4.70 & 149.4 & 149.4 & 149.4 & 149.4 \\
\hline & Med & 728.10 & 4.50 & 294.10 & 149.4 & 149.4 & 149.4 \\
\hline & High & 1103 & 0.75 & 664.70 & 22.63 & 22.63 & 22.63 \\
\hline \multirow{3}{*}{$\begin{array}{c}\text { Erosion rate } \\
\text { density } \\
\text { (E) } \mathbf{x 1 0}^{-5} \mathrm{kgs}^{-} \\
{ }^{1} \mathbf{m}^{-2}\end{array}$} & Low & 3.23 & 7.13 & 6.23 & 6.23 & 6.23 & 7.14 \\
\hline & Med & 3.93 & 8.23 & 6.23 & 6.23 & 6.23 & 7.14 \\
\hline & High & 4.34 & 11.32 & 7.34 & 7.34 & 7.34 & 9.83 \\
\hline
\end{tabular}

\subsection{Sediments Particles Analysis including Layers of Sediment Particles}

Sediment particles are of three types including sand, silt and clay which are present in the reservoir bed of the Tarbela dam in variable proportions in the form of layers.

The bottom layer particles are known as bed flow particles and in this layer flow is such that the sediment particles remain attached to the surface of the river bed with only a slow translational movement. This type of particle flow is present in the reservoir bed, but as sediment delta is moving ahead, this type of flow can be observed at the tunnels surface in the future. Due to the highest density of the sand particles, their concentration is the maximum in this layer. Second layer of sediment particles is the one in which particles interact with each other in jumping fashion and so as known jumping particles. The translational as well as vibrational and rotational motions are present. This type of layer is mainly present in reservoir bed but it is also observed in tunnel to some extent. There is another layer in which particles are suspended in water, with extremely less interaction with the flow field and other particles. Due to small concentration of sediment particles the flow is one way coupled. Concentration of clay particles is maximum in this layer because of lowest density.

The analysis for the composition, density, colour and size of the sediments particle is done using Scanning Electron Microscope (SEM) and Particle Analyzer as shown in Fig. 13. Results are presented in Table 10 for comparison with data provided by WAPDA [17]. 
The particles are found to be circular in shape with an average particle diameter of $\mathrm{d}_{\mathrm{p}}=74.13 \mu \mathrm{m}$ and an average density of $1345 \mathrm{~kg} / \mathrm{m}^{3}$.

A calibration study was first undertaken to ascertain the actual concentration (by volume and by mass) in tunnel. The sample preparation and analysis are shown in Fig. 14 and results are given in Table 11.

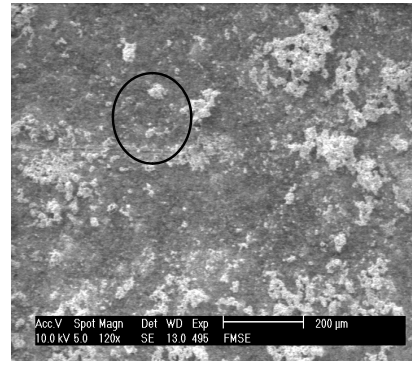

(a)

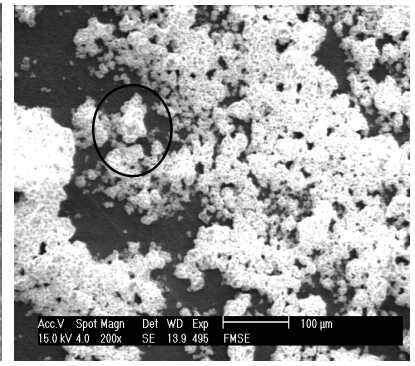

(b)

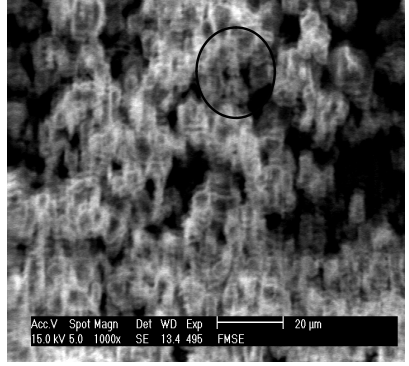

(c)

Fig. 13: SEM Analysis of (a) Sand, (b) Silt, (c) Clay.

Table 10: Sediments particle analysis.

\begin{tabular}{|c|c|c|c|c|c|}
\hline \multirow[b]{2}{*}{$\begin{array}{l}\text { Sediment } \\
\text { particles }\end{array}$} & \multirow{2}{*}{$\begin{array}{c}\text { Sediments } \\
\text { composition } \\
\text { (Jun-Sep) 2008 }\end{array}$} & \multirow[b]{2}{*}{ Density } & \multirow[b]{2}{*}{ Color } & \multicolumn{2}{|c|}{ Particulate diameter, $d_{p}(\mu \mathrm{m})$} \\
\hline & & & & $\begin{array}{c}\text { WAPDA } \\
\text { (1974) }\end{array}$ & $\begin{array}{l}\text { Sample Analysis } \\
\text { in March } 2010\end{array}$ \\
\hline Sand & 31.09 & 1535 & dark grey & 200 & 183 \\
\hline Silt & 53.34 & 1330 & light grey & 40 & 37 \\
\hline Clay & 15.58 & 1170 & light brown & 2 & 2.4 \\
\hline
\end{tabular}

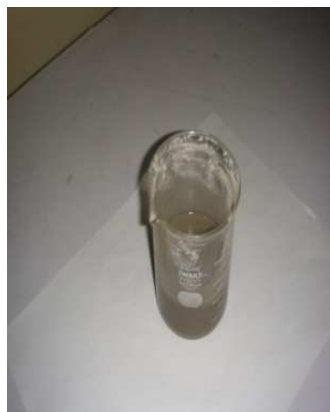

(a)

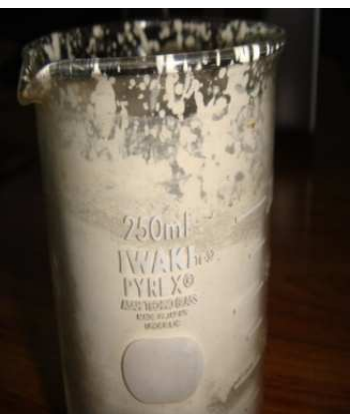

(b)

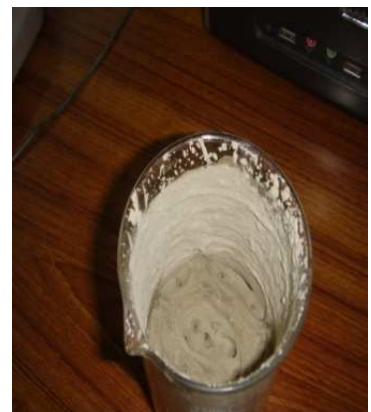

(c)

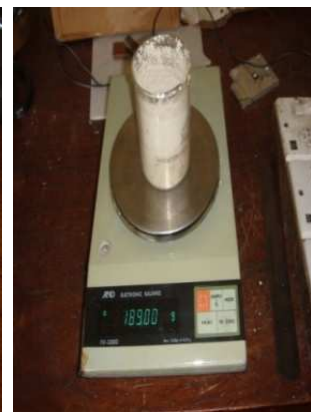

(d)

Fig. 14: (a) Sediments suspension, (b) Heating, (c) Evaporated form, (d) Particulate form. 
Table 11: Sediments particle concentration.

\begin{tabular}{|c|c|}
\hline Mass basis & $21 \%$ \\
\hline Volume basis & $25 \%$ \\
\hline
\end{tabular}

\section{EXPERIMENTAL VALIDATION}

\subsection{Experimental Set-up and Procedure}

An experimental setup is developed to validate the numerical results discussed in section 5.2. The experimental setup is as shown in Fig. 15. The pipe loop is constructed in the horizontal plane with a valve to allow flow to be diverted to another loop as necessary. A high power stirrer is installed to help distribute the sediments in the tank. Pipe components are made of AISI 304L stainless steel with a nominal wall thickness of $3 \mathrm{~mm}$. The geometric components like straight portion, bend-section and T-section are all analysed for their flow characteristics in this study. Pipe sections are prepared for weighing by firstly thoroughly rinsing with water to remove any sediments and then cleaned with warm 5\% citric acid to remove calcite deposits on internal surfaces. This was necessary to allow the change in mass of the pipe sections to be attributed solely to erosion. The pipes are then allowed to air dry, usually overnight, prior to weighing. The experiment is performed for continuous flow of sediments for 80 hours with sediments concentration of about $0.4 \%$ by volume and a velocity of $0.175 \mathrm{~m} / \mathrm{s}$. The Reynolds number calculated is 2997 for this flow. The loops are then carefully dismantled for cleaning and weighing.

\subsection{Numerical Solution of the Experimental Set-up}

Pro-Engineer Wildfire 4.0 is used for the modeling of the components, i.e, straight portion, bend-section and T-section The ANSYS ICEM package is used for the meshing of the geometry. The number of elements used in the geometric components are 1500, 130 and 160 respectively. The analysis is done in ANSYS CFX as shown in Fig. 15. Table 12 shows the input parameters used in the software. Specifications for different regions of Tunnel 3 are summarized in Table 13.

The comparisons between the experimental and numerical results are tabulated in Table 14. The results show an error of about $8 \%$ for erosion rate density.

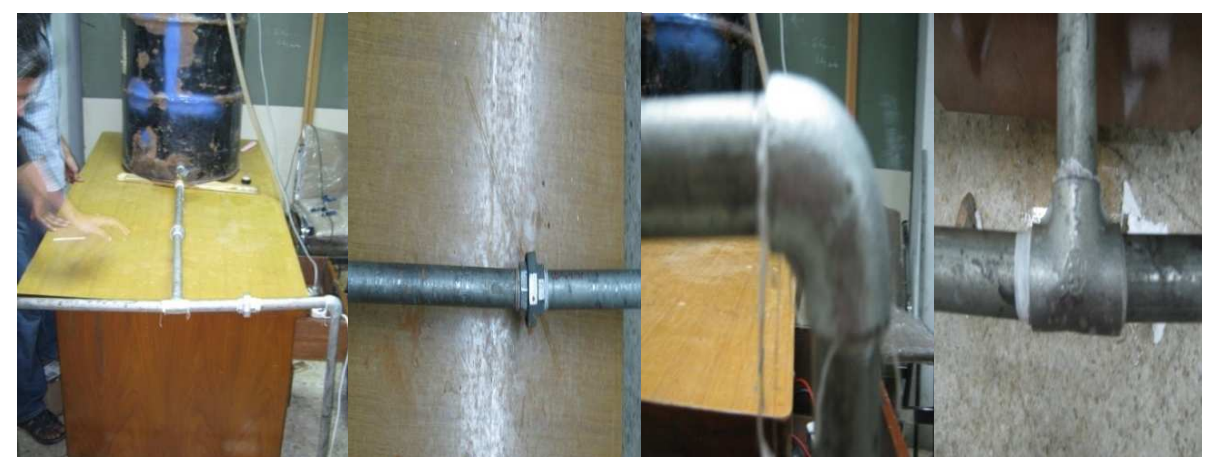


(a)

(b) (c)

(d)

Fig. 15: (a) Complete experimental set-up, (b) Straight portion, (c) Bend section, (d) Tsection

Table 12: Input parameters for the similar geometric components.

\begin{tabular}{|l|l|}
\hline \multicolumn{1}{|c|}{ Parameter } & \multicolumn{1}{c|}{ Value } \\
\hline Radius of Pipe/T/Elbow & $9.525 \times 10^{-3} \mathrm{~m}$ \\
\hline Cross-sectional Area of Pipe/T/Elbow & $2.850 \times 10^{-4} \mathrm{~m}^{2}$ \\
\hline Density of water & $998 \mathrm{~kg} / \mathrm{m}^{3}$ \\
\hline Volume of water & $0.10402 \mathrm{~m}^{3}$ \\
\hline Mass of water & $103.812 \mathrm{Kg}$ \\
\hline Mass of Sediments & $0.4 \mathrm{Kg}$ \\
\hline
\end{tabular}

Table 13: Specifications for different regions of Tunnel 3.

\begin{tabular}{|l|c|c|c|c|}
\hline & \multirow{2}{*}{ Straight Portion } & \multirow{2}{*}{ Bend Section } & \multicolumn{2}{|c|}{ T-Section } \\
\cline { 4 - 5 } & $2.736 \times 10^{-2}$ & $5.428 \times 10^{-3}$ & $5.963 \times 10^{-3}$ \\
\hline Surface Area $\left(\mathrm{m}^{2}\right)$ & $5.00 \times 10^{-5}$ & $5.0010^{-5}$ & $1.0010^{-4}$ & $5.00 \times 10^{-5}$ \\
\hline $\begin{array}{l}\text { Volumetric Flow } \\
\text { Rate }\left(\mathrm{m}^{3} / \mathrm{s}\right)\end{array}$ & 0.175 & 0.175 & 0.350 & 0.175 \\
\hline Velocity (m/s) & 0.0499 & 0.0499 & 0.0998 & 0.0499 \\
\hline $\begin{array}{l}\text { Mass Flow Rate of } \\
\text { Water (m/s) }\end{array}$ & $1.923 \times 10^{-4}$ & $1.923 \times 10^{-4}$ & $3.845410^{-4}$ & $1.92310^{-4}$ \\
\hline $\begin{array}{l}\text { Mass Flow Rate of } \\
\text { Sediments }\left(\mathrm{m}^{3} / \mathrm{s}\right)\end{array}$ & 4.98 & 7.45 & 11.01 & \\
\hline Change in Mass $(\mathrm{g})$ & & & \multicolumn{2}{|c|}{} \\
\hline
\end{tabular}

Table 14: Comparison between Experimental and Numerical result.

\begin{tabular}{|l|c|c|c|}
\hline & Straight Portion & Bend Section & T-Section \\
\hline $\begin{array}{l}\text { Erosion Rate } \\
\left(\mathrm{kg} / \mathrm{m}^{2} . \mathrm{s}\right)\end{array}$ & $6.319 \times 10^{-7}$ & $4.766 \times 10^{-6}$ & $6.411 \times 10^{-6}$ \\
\hline $\begin{array}{l}\text { Erosion Rate } \\
\text { from CFX } \\
\left(\mathrm{kg} / \mathrm{m}^{2} . \mathrm{s}\right)\end{array}$ & $6.909 \times 10^{-7}$ & $4.960 \times 10^{-6}$ & $6.638 \times 10^{-6}$ \\
\hline$\%$ difference & 8.50 & 3.90 & 3.40 \\
\hline
\end{tabular}




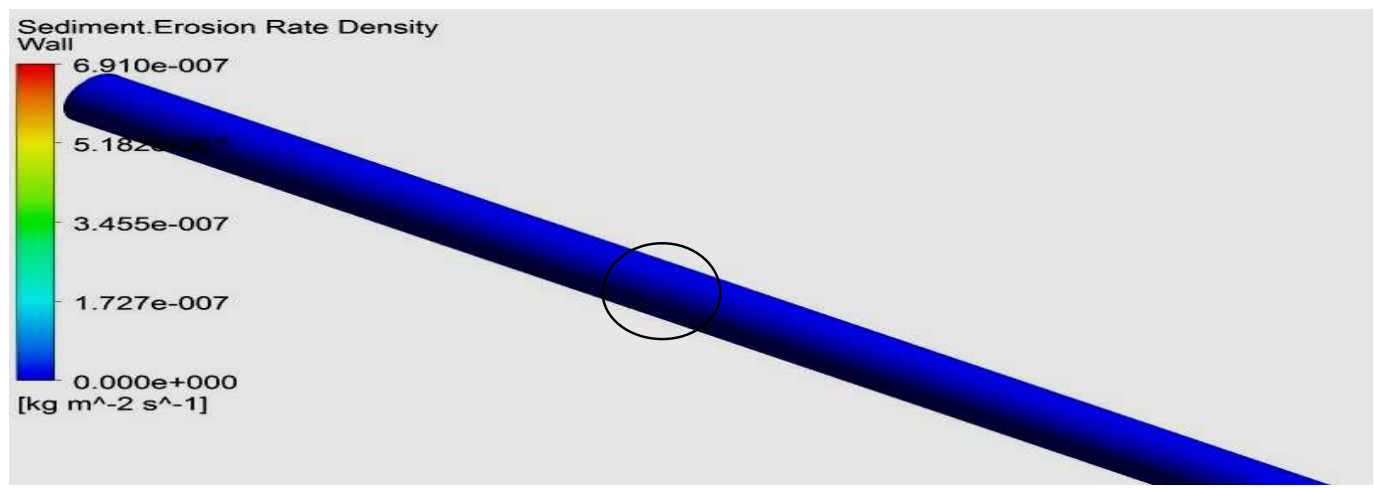

(a)

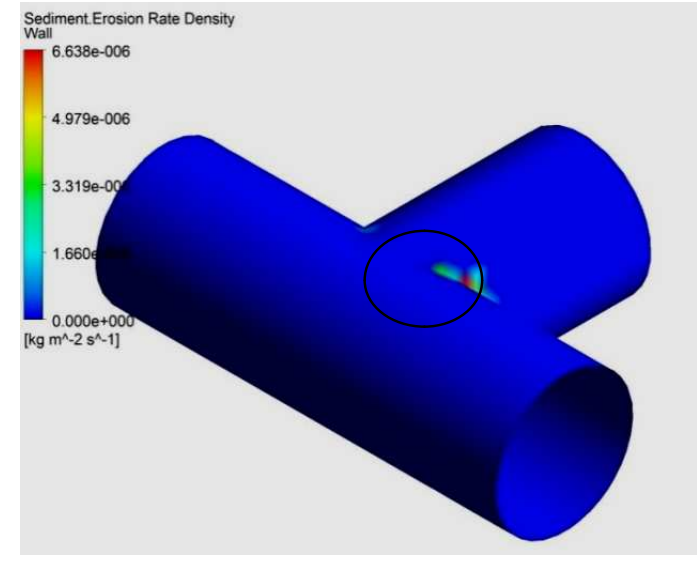

(b)

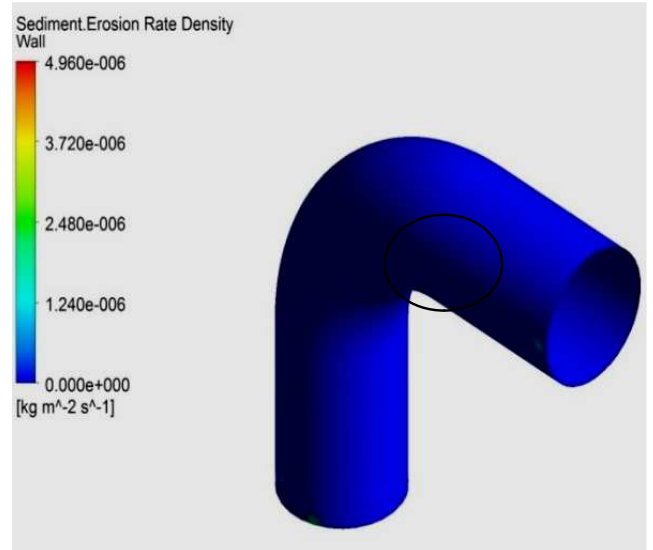

(c)

Fig. 15: Erosion rate density profiles for the: (a) Straight portion, (b) T-section, (c) Bend-section.

\section{CONCLUSION}

A CFD-based velocity, pressure and erosion prediction procedure is presented, based on one way and two way/full coupling phenomena. We have found that erosion is high at high head because of higher impact velocity of the sediment particles during July, August and September. Moreover, the results are shown for nine different locations of critical importance with and without sediment particles flowing through the tunnel where the velocities and pressures may vary causing the erosive damage. Interestingly, the erosion rate density is found to be maximum at the S-bend due to the additive effect of the cavitation erosion. Also, higher values of erosion are observed at the outlet branches due to several reasons like the higher velocity and impact angle and the production of turbulent eddies. Furthermore, the cavitation phenomenon is observed at the S-bend due to the lower local pressure then the vapor pressure at this critical location. Numerical simulations as well as experimental erosion tests are performed. Comparisons show that the CFD-based erosion prediction procedure is able to reasonably predict the erosion profile and satisfactorily capture the trend of erosion with respect to the carrier velocity with a 
maximum error of $8.5 \%$. Simulation results show that particle volume fraction increases rapidly as the sediment delta moving towards the tunnels. The particle concentration is found to be $21 \%$ by mass and $21 \%$ by volume. This increase in particle concentration is changing continuous flow to dispersed flow and is damaging the tunnel walls. The particulate analysis is shown to have a good agreement with the data provided by WAPDA (water and power development authority). The error in the average diameter of the particles found to be only $7.8 \%$.

\section{ACKNOWLEDGEMENT}

The authors acknowledge Pak-US project, WAPDA and UIUC USA in completing this study. The authors also acknowledge the support and working of Mr Ahmed Danial, Mr. Talal Mahmood, Mr. Abdul Muqeet Haider and Mr. Azeem Sarwar in completing this extensive study.

\section{REFERENCES}

[1] M. Hanif, Sediment Concentration (ppm). Annual Reservoir Sedimentation report, Survey and Hydrology Department, Tarbela Dam Project. pp. 5. 2009.

[2] Cunkui Huang, S. Chiovelli, P. Minev, Jingli Luo and K. Nandakumar. A comprehensive phenomenological model for erosion of materials in jet flow. Powder Technology, (187), 273-279, 20 November, 2008.

[3] A. Hossain, J. Naser, K. Macmanus, G. Ryan. CFD Investigation of particle deposition and distribution in a horizontal pipe. Third International Conference on CFD in the process industries. CSIRO, Melbourne, Australia. pp. 489-492.10-12, December 2006.

[4] C. Xianghui, S.M. Brenton, A.S. Siamack, Application and experimental validation of a computational fluid dynamics (CFD)-based erosion prediction model in elbows and plugged tees, Computers \& Fluids (33), 1251-1272. 2004.

[5] S. Dosanjh and J.A.C Humphrey. The influence of turbulence on erosion by a particle laden fluid jet, Wear (102), 309-330. 1985.

[6] G. Brown. Use of CFD to predict and reduce erosion in industrial slurry piping system. Fifth International Conference on CFD in the process industries. CSIRO, Melbourne, Australia, pp. 13-15. December 2006.

[7] H. Prasad Neopane. Faculty of Engineering Science and Technology, Norwegian University of Science and Technology (NTNU) in partial fulfillment of the requirement of the degree of Doctor of Philosophy. Trondheim, Norway, March 2010.

[8] Drag models. ANSYS CFX Solver Theory Guide, Release 11.0. pp. 2. (19962006). 
[9] L. Bouhadji, Three Dimensional Numerical Simulation of Turbulent Flow Over Spillways, ASL-AQFlow Inc., Sidney, British Columbia, Canada. pp 1-7. 2003.

[10] B. Dobrowolski and J. Wydrich. Evaluation of Numerical models for prediction of areas subjected to erosive. Int. J. of Applied Mechanics and Engineering (11), 735-749. 2006.

[11] ANSYS CFX Reference Guide, Release 11.0. pp. 1-4. (1996-2006).

[12] B.R. Munson, T.H. Okiishi, Fundamentals of Fluid Mechanics, 5th Edition. ISBN 9812-53-221-8. 2006.

[13] Erosion models. ANSYS CFX Solver Theory Guide, Release 11.0. pp. 1- 2. (19962006).

[14] Jinyu Jiao, Zhengliang Liu and Ying Zheng. Evaluations and Modifications on Reynolds Stress Model in Cyclone Simulations, Chem. Eng. Technol., (30), 1520, 2007.

[15] Tippets and Abbett. Civil Engineering Works. Contract documents for construction. Tarbela Dam Project. TAMS, International (6), p. 16. 1967. Dam Project. pp. 7-8. 2000.

[16] P. J. Zwart, A. G. Gerber, and T. Belamri. A Two-Phase Flow Model for Predicting Cavitation Dynamics. ICMF 2004 International Conference on Multiphase Flow. Yokohama, Japan, May 30-June 3, 2004. Paper No.152.

[17] Sediments composition. Annual Reservoir Sedimentation report, Survey and Hydrology Department, Tarbela Dam Project. pp. 10-11. 2008. 


\section{NOMENCLATURE}

\begin{tabular}{|c|c|}
\hline$B$ & body force due to sediment particles \\
\hline $\mathrm{Ca}$ & cavitation number \\
\hline$C_{D}$ & drag coefficient \\
\hline $\mathrm{C}_{\mu}$ & anisotropic Diffusion Coefficient \\
\hline$C_{s 1}, C_{s 2,} C_{1 \varepsilon,} C_{2 \varepsilon}$ & RS Coefficients \\
\hline$d_{p}$ & particle diameter \\
\hline E & erosion rate density \\
\hline$F_{D}$ & drag force \\
\hline $\mathrm{F}_{\text {cond }}$ & cavitation condensation coefficient \\
\hline $\mathrm{F}_{\text {vap }}$ & cavitation vaporization coefficient \\
\hline$g$ & gravitational acceleration of the sediment particles \\
\hline$k$ & erosion model constant \\
\hline$m$ & particle mass \\
\hline$n$ & velocity exponent \\
\hline $\mathrm{P}_{\mathrm{s}}$ & saturation pressure of water \\
\hline$p$ & fluid pressure \\
\hline $\mathrm{p}_{\mathrm{v}}$ & vapour pressure \\
\hline $\mathrm{R}_{\mathrm{B}}$ & bubble radius \\
\hline $\operatorname{Re}_{\mathrm{p}}$ & particle Reynolds number \\
\hline $\mathrm{R}_{\mathrm{f}}$ & cavitation rate under relaxation factor \\
\hline$\square_{f}$ & fluid phase volume fraction \\
\hline$r_{\text {nuc }}$ & nuclei volume fraction \\
\hline$\square_{p}$ & particulate phase volume fraction \\
\hline$U_{c}$ & fluid velocity \\
\hline
\end{tabular}




$$
\begin{array}{ll}
U_{p} & \text { particle velocity } \\
U & \text { particle relative velocity } \\
V_{p} & \text { particle impact velocity }
\end{array}
$$

$\begin{array}{cl}\text { Greek letters } & \\ \beta & \text { particle mass loadings } \\ \gamma & \text { particle impact angle } \\ \rho_{f} & \text { fluid density } \\ \rho_{p} & \text { particle density } \\ \rho_{\mathrm{s}} & \text { nucleation site density } \\ \rho_{\mathrm{r}} & \text { density ratio } \\ \sigma & \text { stress tensor } \\ \sigma_{\varepsilon} & \text { turbulence model constant } \\ \sigma_{\mathrm{k}} & \text { turbulence model constant for the k-equation } \\ \sigma_{\mathrm{t}} & \text { surface tension coefficient } \\ \tau_{p} & \text { particle response time } \\ \mu & \text { fluid dynamic viscosity } \\ v & \text { fluid kinematic viscosity }\end{array}$

